\title{
AN APPARATUS FOR THE PHOTOGRAPHIC RECORDING OF EYE MOVEMENTS.
}

\author{
By M. D. VERNON. \\ (From the Cambridge Psychological Laboratory.)
}

THE apparatus described in this paper is based upon a modification of Dodge's method of recording eye movements photographically'. By means of the former, a record is obtained upon a continuously moving film of the movements of a bright spot of light reflected from the cornea. It has been shown by Dodge ${ }^{2}$ that the movements of such a spot are the same in direction as those of the eyeball itself, but are only about half as great in extent. Fig. 1 shows the apparatus viewed from the front, and Fig. 2 shows it in section along a vertical plane, parallel to the sagittal plane, passing through the subject's right eye.

The source of light is a 100 c.P. Ediswan Pointolite bulb; the light is emitted from a small very bright ball, which forms an excellent point source. The light from the bulb $D$ (Fig. 1), contained in the brass chamber $C$, passes through a blue gelatin filter and the narrow aperture, $3 \mathrm{~mm}$. in diameter, of a diaphragm placed at $J$. The divergent beam of blue light passes along the brass tube $T_{1}$, impinges on the right-angled prism $P_{1}$, and is totally reflected on to the cornea of the subject's right eye, as shown in Fig. 2. The beam is reflected by the cornea in a direction parallel to the sagittal plane, at an angle of about $50^{\circ}$ with the horizontal; and impinges on a second right-angled prism $P_{2}$, by which it is refracted and totally reflected into the brass tube $T_{2}$ of the camera $M$. The divergent beam is focussed by the lens $L$, a Ross, London, Xpres camera lens of aperture $1: 4.5$ and focal length $4 \frac{3}{8}$ in., and after passing through the tube $T_{2}$ and the aperture of the camera at $A$, forms an image on the film at $F_{1}$. In Fig. 2 the camera is shown in position for a vertically moving film. The latter passes from the film spool $F_{2}$ over the two pulleys $O_{1}$ and $O_{2}$, and is attached to the heavy brass pulley $O_{3}$. The drive is conveyed from a motor and reducing gear to the fourth pulley $O_{4}$, the shaft of which engages with the shaft of $O_{2}$ by means of a worm gear. Thus the film travels smoothly downwards past the aperture $A$, and is wound on to the pulley $O_{3}$. In these experiments it was driven at a

\footnotetext{
1 Dodge, R. and Cline, T. S., Psychol. Rev. 1901, virr, 145.

2 Dodge, R., Psychol. Rev. Monog. 1907, viII, No. 4.
} 

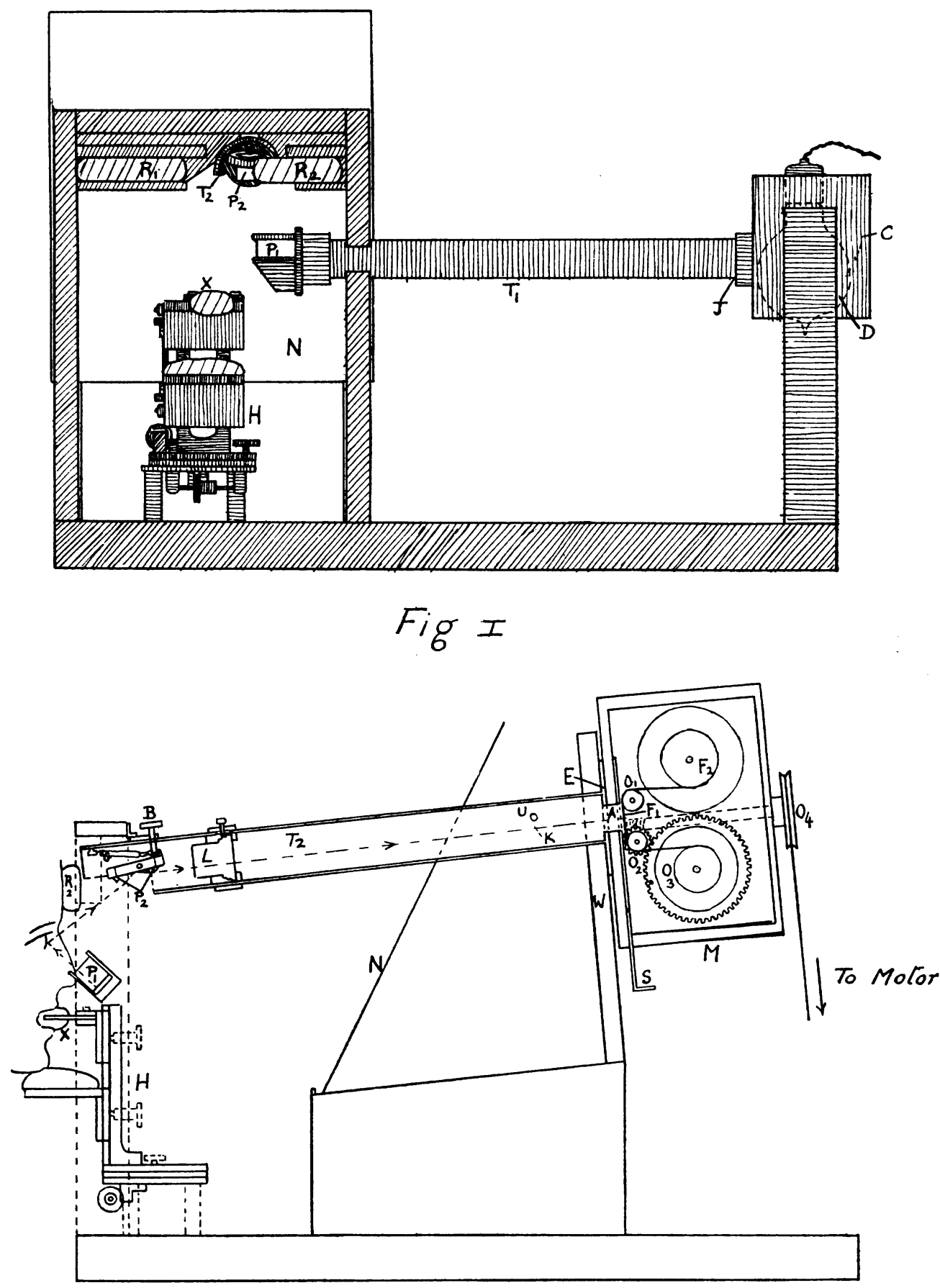

Fig II

J. of Psych. xxı. 1 


\section{Apparatus for Photographic Recording of Eye Movements}

speed of approximately $0.5 \mathrm{~cm}$. per second. The aperture can be closed by means of a shutter, $S$, which slides in and out.

The experimental procedure is as follows. The subject places his head in position on the head-rest, $H$, with his chin on the chin-rest and his forehead pressed against the two adjustable pads $R_{1}$ and $R_{2}$. He grips with his teeth a piece of dentist's wax $X$; an impression of his teeth is taken, so that they can be held firmly in the same position throughout the experiments. Both the mouth and chin-rests can be adjusted vertically; and the head-rest can be moved as a whole in two horizontal directions at right angles to each other. By turning the tube $T_{1}$ about its axis, the beam of light is directed to form a bright spot of light on the cornea. The angle at which it falls on the cornea is such that, when the eye looks straight forwards, the beam does not enter the pupil, and is thus not visible to the subject. The reflected beam is directed along the tube $T_{2}$ by rotating the prism $P_{2}$ about its horizontal axis. The rotation is effected by raising or lowering the screw-head $B$; the prism is kept tightly pressed against the screw by means of the spring $G$. If necessary, the position of the head is also adjusted. The camera is previously removed from the upright $W$, and a piece of ground glass at the end of a tube placed in the position normally occupied by the film; the reflected image is then focussed on the ground glass by sliding the lens $L$ towards or away from the camera. The latter is then replaced, the motor started and the shutter opened. A photographic record is thus obtained of the movements of the eyeball, made either voluntarily or in reading. The reading material, or a sheet of paper bearing a series of points to be fixated, is placed in front of the subject against the screen $N$.

The camera is held steadily in position by flanges fixed to a metal plate $E$, which in turn is screwed to the wooden upright $W$. If the screws are removed, the camera and plate can be swivelled about the tube $T_{2}$ until the camera and film are horizontal, and the metal plate again screwed to $W$. With a vertically moving film, a record of the horizontal components of the eye movements is obtained, and with a horizontally moving film, a record of the vertical components.

A time signal is used for measuring the exact speed of movement of the film. A narrow convergent beam of light is directed through the aperture $U$ on to a small piece of plane glass $K$ situated on the right-hand side (facing the subject) of the tube $T_{2}$. The light is reflected by this piece of glass to form an image at the right-hand edge of the vertically moving film. A time marker (not shown) placed outside $T_{2}$ in the path 
of the beam allows the light to flash once a second on to the film, thus giving a time record which does not interfere with the eye-movement record. This time signal can only be utilized with the vertically moving film.

This apparatus has been found to work quite satisfactorily, and a number of records have been obtained both of eye movements made voluntarily and of those made in normal reading. Fixations appear as fine black lines on the film. The speed of movement of the film is sufficient to show clearly the fixations and re-fixations which occur in normal reading. Voluntary movements up to $40^{\circ}$ of arc can be recorded; they cover about $1 \mathrm{~cm}$. on the film. By use of magnification, movements of $1^{\circ}$ of arc and less are readily measurable.

In conclusion I wish to express my gratitude to Prof. Hartridge for his advice and help in designing this apparatus, and to Dr E. A. Schuster for constructing it at the National Institute of Medical Research, Hampstead; and to thank the Medical Research Council for permission to use the accompanying figures.

(Manuscript received 20 December, 1929.) 